\title{
REVERSIBILITY OF DISCONNECTED STRUCTURES
}

\author{
Miloš S. Kuriliợ 1 and Nenad Morača
}

\begin{abstract}
A relational structure is called reversible iff every bijective endomorphism of that structure is an automorphism. We give several equivalents of that property in the class of disconnected binary structures and some its subclasses. For example, roughly speaking and denoting the set of integers by $\mathbb{Z}$, a structure having reversible components is reversible iff its components can not be "merged" by condensations (bijective homomorphisms) and each $\mathbb{Z}$ sequence of condensations between different components must be, in fact, a sequence of isomorphisms. We also give equivalents of reversibility in some special classes of structures. For example, we characterize CSB linear orders of a limit type and show that a disjoint union of such linear orders is a reversible poset iff the corresponding sequence of order types is finite-to-one.

2010 Mathematics Subject Classification: 03C07, 03E05, 05C40, 06A06.

Keywords: binary relation, disconnected structure, reversibility, partial order.
\end{abstract}

\section{Introduction}

A relational structure $\mathbb{X}$ is said to be reversible iff every bijective endomorphism $f: \mathbb{X} \rightarrow \mathbb{X}$ is an automorphism and the relevance of that property follows from the fact that the class of reversible structures includes linear orders, Boolean lattices, well founded posets with finite levels [2, 3], tournaments, Henson graphs [9], and Henson digraphs [5]. In addition, reversibility is an invariant of some forms of biinterpretability [5], extreme elements of $L_{\infty \omega}$-definable classes of structures are reversible under some syntactical restrictions [9], and all structures first-order definable in linear orders by quantifier-free formulas without parameters (i.e., monomorphic or chainable structures) are reversible [6].

In this article we investigate reversibility in the class of binary structures, that is models of the relational language $L_{b}=\langle R\rangle$, where $\operatorname{ar}(R)=2$, and, moreover, we restrict our attention to the class of disconnected $L_{b}$-structures. (If $\mathbb{X}=\langle X, \rho\rangle$ is an $L_{b}$-structure and $\sim_{\rho}$ the minimal equivalence relation on $X$ containing $\rho$, then the corresponding equivalence classes are called the connectivity components of $\mathbb{X}$ and $\mathbb{X}$ is said to be disconnected if it has more than one component, that is, if $\sim_{\rho} \neq X^{2}$ ). The prototypical disconnected structures are, of course, equivalence

\footnotetext{
${ }^{1}$ Department of Mathematics and Informatics, Faculty of Science, University of Novi Sad, Trg Dositeja Obradovića 4, 21000 Novi Sad, Serbia. email: milos@dmi.uns.ac.rs

${ }^{2}$ Department of Mathematics and Informatics, Faculty of Science, University of Novi Sad, Trg Dositeja Obradovića 4, 21000 Novi Sad, Serbia. email: nenad.moraca@dmi.uns.ac.rs
} 
relations themselves; other prominent representatives of that class are some countable ultrahomogeneous graphs and posets (see [13, 18]), non-rooted trees, etc.

By [10], a disconnected $L_{b}$-structure $\mathbb{X}=\bigcup_{i \in I} \mathbb{X}_{i}$ belonging to a wide class $\mathrm{RFM}^{3}$ of structures (containing equivalence relations) is reversible iff the sequence of cardinalities of its connectivity components, $\left\langle\left|X_{i}\right|: i \in I\right\rangle$, has the following property: a sequence of non-zero cardinals $\left\langle\kappa_{i}: i \in I\right\rangle$ is defined to be reversible iff

$$
\neg \exists f \in \operatorname{Sur}(I) \backslash \operatorname{Sym}(I) \quad \forall j \in I \quad \sum_{i \in f^{-1}[\{j\}]} \kappa_{i}=\kappa_{j},
$$

where $\operatorname{Sym}(I)$ (resp. $\operatorname{Sur}(I)$ ) denotes the set of all bijections (resp. surjections) $f: I \rightarrow I$. For the following characterization of such sequences we recall that a set $K \subset \mathbb{N}$ is called independent iff $n \notin\langle K \backslash\{n\}\rangle$, for all $n \in K$, where $\langle K \backslash\{n\}\rangle$ is the subsemigroup of the semigroup $\langle\mathbb{N},+\rangle$ generated by $K \backslash\{n\}$; by $\operatorname{gcd}(K)$ we denote the greatest common divisor of the numbers from $K$. By [10] we have

Fact 1.1 A sequence of non-zero cardinals $\left\langle\kappa_{i}: i \in I\right\rangle$ is reversible if and only if either it is a finite-to-one sequence, or it is a sequence of natural numbers, the set $K=\left\{m \in \mathbb{N}: \kappa_{i}=m\right.$, for infinitely many $\left.i \in I\right\}$ is non-empty and independent, and $\operatorname{gcd}(K)$ divides at most finitely many elements of the set $\left\{\kappa_{i}: i \in I\right\}$.

In Section 2 we give several equivalents of reversibility in the class of disconnected $L_{b}$-structures and here we describe one of them. First, by Theorem 2.1, the components of a reversible structure must be reversible and, hence, the following assumption (containing that restriction) will appear in several parts of the text:

(*) $\mathbb{X}_{i}=\left\langle X_{i}, \rho_{i}\right\rangle, i \in I$, are pairwise disjoint, connected and reversible $L_{b^{-}}$ structures, $\mathbb{X}=\bigcup_{i \in I} \mathbb{X}_{i}$ and $\mathcal{X}=\left\{\mathbb{X}_{i}: i \in I\right\}$.

Second, generalizing (1) and writing $\mathbb{X} \preccurlyeq_{c} \mathbb{Y}$ iff there is a bijective homomorphism $g: \mathbb{X} \rightarrow \mathbb{Y}$, we will say that a sequence of $L_{b}$-structures $\left\langle\mathbb{X}_{i}: i \in I\right\rangle$ is a reversible sequence of structures iff

$$
\neg \exists f \in \operatorname{Sur}(I) \backslash \operatorname{Sym}(I) \quad \forall j \in I \quad \bigcup_{i \in f^{-1}[\{j\}]} \mathbb{X}_{i} \preccurlyeq c \mathbb{X}_{j}
$$

Third, assuming (*), a mapping $i: \mathbb{Z} \rightarrow I$, usually denoted by $\left\langle i_{k}: k \in \mathbb{Z}\right\rangle$, will be called a $\mathbb{Z}$-sequence in $I$ iff it is an injection and

$$
\forall k, l \in \mathbb{Z}\left(k<l \Rightarrow \mathbb{X}_{i_{k}} \preccurlyeq{ }_{c} \mathbb{X}_{i_{l}}\right),
$$

\footnotetext{
${ }^{3} \mathrm{RFM}$ is the class of structures $\mathbb{X}=\bigcup_{i \in I} \mathbb{X}_{i}$ such that $\left\langle\mathbb{X}_{i}: i \in I\right\rangle$ is a sequence of pairwise disjoint, connected and reversible $L_{b}$-structures which is rich for monomorphisms, which means that for all $i, j \in I$ and each $A \in\left[X_{j}\right]^{\left|X_{i}\right|}$ there is a monomorphism $g: \mathbb{X}_{i} \rightarrow \mathbb{X}_{j}$ such that $g\left[X_{i}\right]=A$.
} 
which is, by the transitivity of the relation $\preccurlyeq_{c}$, equivalent to the existence of a sequence of condensations $g_{k}: \mathbb{X}_{i_{k}} \rightarrow \mathbb{X}_{i_{k+1}}$, for $k \in \mathbb{Z}$. If, in addition, $\mathbb{X}_{i_{k}} \cong \mathbb{X}_{i_{l}}$, for all $k, l \in \mathbb{Z}$, the $\mathbb{Z}$-sequence $\left\langle i_{k}: k \in \mathbb{Z}\right\rangle$ will be called trivial $!^{4}$

The announced equivalent of reversibility is given in the following theorem.

Theorem $1.2(*)$ The structure $\mathbb{X}$ is reversible iff $\left\langle\mathbb{X}_{i}: i \in I\right\rangle$ is a reversible sequence of structures and each $\mathbb{Z}$-sequence in I is trivial ${ }^{5}$

In Section 2, in addition, we give equivalents of reversibility in some special classes of structures (structures having finitely many components, structures with finite components, disjoint unions of linear orders and tournaments).

In Sections 3 and 4 we give some sufficient conditions for reversibility of disconnected structures and detect several classes of reversible partial orders. In Section 5 we apply these results to unions of disjoint linear orders. In particular, we characterize CSB linear orders of a limit type and show that a disjoint union of such linear orders, $\bigcup_{i \in I} \mathbb{X}_{i}$, is a reversible poset iff $\left\langle\operatorname{otp}\left(\mathbb{X}_{i}\right): i \in I\right\rangle$ is a finiteto-one sequence. Similarly, a disjoint union of $\sigma$-scattered linear orders, $\bigcup_{i \in I} \mathbb{X}_{i}$, is reversible if $\left\langle\left[\mathbb{X}_{i}\right]_{\rightleftarrows}: i \in I\right\rangle$ is a finite-to-one sequence, where $\left[\mathbb{X}_{i}\right]_{\rightleftarrows}$ is the equimorphism (bi-embedability) class of $\mathbb{X}_{i}$ in $\mathcal{X}$.

In the rest of this section we introduce notation and recall basic facts which will be used in the paper.

Condensational order, equivalence and reversibility $\quad$ If $\mathbb{X}$ and $\mathbb{Y}$ are $L_{b}$-structures, $\operatorname{Iso}(\mathbb{X}, \mathbb{Y}), \operatorname{Cond}(\mathbb{X}, \mathbb{Y}), \operatorname{Mono}(\mathbb{X}, \mathbb{Y})$ and $\operatorname{Emb}(\mathbb{X}, \mathbb{Y})$ will denote the set of all isomorphisms, condensations (bijective homomorphisms), monomorphisms and embeddings from $\mathbb{X}$ to $\mathbb{Y}$ respectively. $\operatorname{Iso}(\mathbb{X}, \mathbb{X})=\operatorname{Aut}(\mathbb{X})$ is the set of automorphisms of $\mathbb{X}$, instead of $\operatorname{Cond}(\mathbb{X}, \mathbb{X})$ we will write $\operatorname{Cond}(\mathbb{X})$ etc.

The condensational preorder $\preccurlyeq_{c}$ on the class of $L_{b}$-structures is defined by $\mathbb{X} \preccurlyeq_{c} \mathbb{Y}$ iff $\operatorname{Cond}(\mathbb{X}, \mathbb{Y}) \neq \emptyset$, the condensational equivalence is the equivalence relation defined on the same class by: $\mathbb{X} \sim_{c} \mathbb{Y}$ iff $\mathbb{X} \preccurlyeq_{c} \mathbb{Y}$ and $\mathbb{Y} \preccurlyeq_{c} \mathbb{X}$, and it determines the antisymmetric quotient of the condensational preorder, the condensational order, in the usual way. We will write $\mathbb{X} \prec_{c} \mathbb{Y}$ iff $\mathbb{X} \preccurlyeq_{c} \mathbb{Y}$ and $\mathbb{Y} \varkappa_{c} \mathbb{X}$ (which is for reversible structures equivalent to $\mathbb{X} \preccurlyeq_{c} \mathbb{Y}$ and $\mathbb{Y} ¥ \mathbb{X}$, see Fact 1.4).

Some simple properties equivalent to reversibility are listed in the following claim (which, in fact, holds for any relational language $L$, see [11]).

\footnotetext{
${ }^{4}$ We note that $\left\langle i_{k}: k \in \mathbb{Z}\right\rangle$ is a $\mathbb{Z}$-sequence in $I$ iff $k \mapsto \mathbb{X}_{i_{k}}$ is a monomorphism from the linear order $\langle\mathbb{Z},<\rangle$ to the preorder $\left\langle\mathcal{X}, \preccurlyeq_{c}\right\rangle$. It is trivial iff all $\mathbb{X}_{i_{k}}$ 's are in the same $\sim_{c}$-class (or, equivalently, $\cong$-class) of that preorder (see Fact 1.4 ).

${ }^{5}$ In other words, the union $\bigcup_{i \in I} \mathbb{X}_{i}$ is not reversible iff the components can be "merged by condensations" or there is a non-trivial $\mathbb{Z}$-sequence in $I$.
} 
Fact 1.3 For an $L_{b}$-structure $\mathbb{X}=\langle X, \rho\rangle$ the following conditions are equivalent

(a) $\mathbb{X}$ is a reversible structure (that is, $\operatorname{Cond}(\mathbb{X})=\operatorname{Aut}(\mathbb{X})$ ),

(b) $\forall \sigma \subset X^{2} \quad(\sigma \varsubsetneqq \rho \Rightarrow\langle X, \sigma\rangle \not\langle X, \rho\rangle)$,

(c) $\forall \sigma \subset X^{2} \quad(\rho \mp \sigma \Rightarrow\langle X, \sigma\rangle \not\langle X, \rho\rangle)$,

(d) $\mathbb{X}^{c}$ is a reversible structure, where $\mathbb{X}^{c}=\left\langle X, \rho^{c}\right\rangle$ and $\rho^{c}=X^{2} \backslash \rho$.

We remark that if $\mathbb{X}$ is a connected binary structure but its complement $\mathbb{X}^{c}$ is disconnected then, by Fact 1.3 , the reversibility of $\mathbb{X}^{c}$ implies the reversibility of $\mathbb{X}$. Thus the results concerning reversibility of disconnected structures can be converted into results about reversibility of such connected structures. (We note that at least one of the structures $\mathbb{X}$ and $\mathbb{X}^{c}$ is connected, see [4])

Reversible structures have the Cantor-Schröder-Bernstein property for condensations. Moreover, for any relational language $L$ we have (see [11])

Fact 1.4 Let $\mathbb{X}$ and $\mathbb{Y}$ be L-structures. If $\mathbb{X}$ is a reversible structure and $\mathbb{Y} \sim_{c} \mathbb{X}$, then $\mathbb{Y} \cong \mathbb{X}$ (thus $\mathbb{Y}$ is reversible too $)$ and $\operatorname{Cond}(\mathbb{X}, \mathbb{Y})=\operatorname{Iso}(\mathbb{X}, \mathbb{Y})$.

Morphisms of disconnected $L_{b}$-structures If $\mathbb{X}=\langle X, \rho\rangle$ is an $L_{b}$-structure, then the transitive closure $\sim_{\rho}$ of the relation $\rho_{r s}=\Delta_{X} \cup \rho \cup \rho^{-1}$ (given by $x \sim_{\rho} y$ iff there are $n \in \mathbb{N}$ and $z_{0}=x, z_{1}, \ldots, z_{n}=y$ such that $z_{i} \rho_{r s} z_{i+1}$, for each $i<n)$ is the minimal equivalence relation on $X$ containing $\rho$. The corresponding equivalence classes $[x], x \in X$, are called the components of $\mathbb{X}$, and the structure $\mathbb{X}$ is called connected iff $\left|X / \sim_{\rho}\right|=1$.

If $\mathbb{X}_{i}=\left\langle X_{i}, \rho_{i}\right\rangle, i \in I$, are connected $L_{b}$-structures and $X_{i} \cap X_{j}=\emptyset$, for different $i, j \in I$, then the structure $\bigcup_{i \in I} \mathbb{X}_{i}=\left\langle\bigcup_{i \in I} X_{i}, \bigcup_{i \in I} \rho_{i}\right\rangle$ is the disjoint union of the structures $\mathbb{X}_{i}, i \in I$, and the structures $\mathbb{X}_{i}, i \in I$, are its components. A proof of the following fact is direct; see [8] for (a) and [4] for (b).

Fact 1.5 Let $\left\{\mathbb{X}_{i}: i \in I\right\}$ and $\left\{\mathbb{Y}_{j}: j \in J\right\}$ be families of pairwise disjoint and connected $L_{b}$-structures and let $\mathbb{X}=\langle X, \rho\rangle$ and $\mathbb{Y}=\langle Y, \sigma\rangle$ be their unions. Then

(a) $F \in \operatorname{Cond}(\mathbb{X}, \mathbb{Y})$ iff $F=\bigcup_{i \in I} g_{i}$, where $f: I \stackrel{\text { onto }}{\rightarrow} J, g_{i} \in \operatorname{Mono}\left(\mathbb{X}_{i}, \mathbb{Y}_{f(i)}\right)$, for $i \in I$, and $\left\{g_{i}\left[X_{i}\right]: i \in f^{-1}[\{j\}]\right\}$ is a partition of $Y_{j}$, for each $j \in J$;

(b) $F \in \operatorname{Emb}(\mathbb{X}, \mathbb{Y})$ iff $F=\bigcup_{i \in I} g_{i}$, where $f: I \rightarrow J, g_{i} \in \operatorname{Emb}\left(\mathbb{X}_{i}, \mathbb{Y}_{f(i)}\right)$, for $i \in I$, and $\left\langle g_{i}(x), g_{i^{\prime}}\left(x^{\prime}\right)\right\rangle \notin \sigma_{r s}$, whenever $i \neq i^{\prime}, x \in X_{i}$ and $x^{\prime} \in X_{i^{\prime}}$.

\section{Reversibility of disconnected $L_{b}$-structures}

In this section we prove Theorem 1.2 and give some other equivalents of reversibility in the class of disconnected $L_{b}$-structures. 
Theorem 2.1 If $\mathbb{X}_{i}, i \in I$, are pairwise disjoint and connected $L_{b}$-structures, then $\bigcup_{i \in I} \mathbb{X}_{i}$ is reversible iff $\bigcup_{i \in J} \mathbb{X}_{i}$ is reversible for each non-empty set $J \subset I$.

Thus, if $\bigcup_{i \in I} \mathbb{X}_{i}$ is reversible, then all components $\mathbb{X}_{i}, i \in I$, are reversible.

Proof. Let $\mathbb{X}_{i}=\left\langle X_{i}, \rho_{i}\right\rangle$, for $i \in I$, and let $\mathbb{X}=\langle X, \rho\rangle=\left\langle\bigcup_{i \in I} X_{i}, \bigcup_{i \in I} \rho_{i}\right\rangle$.

The implication " $\Leftarrow$ " is trivial. If there exist a non-empty set $J \subset I$ and $f \in \operatorname{Cond}\left(\bigcup_{i \in J} \mathbb{X}_{i}\right) \backslash \operatorname{Aut}\left(\bigcup_{i \in J} \mathbb{X}_{i}\right)$, then there are $x, y \in \bigcup_{i \in J} X_{i}$ such that $\langle x, y\rangle \notin \bigcup_{i \in J} \rho_{i}$ and $\langle f(x), f(y)\rangle \in \bigcup_{i \in J} \rho_{i}$. Now, $F:=f \cup \operatorname{id}_{\bigcup_{i \in I \backslash J} X_{i}} \in$ $\operatorname{Sym}(X)$, it is easy to check that $F \in \operatorname{Cond}(\mathbb{X})$, and the pair $\langle x, y\rangle$ witnesses that $F \notin \operatorname{Aut}(\mathbb{X})$. So $\mathbb{X}$ is not a reversible structure.

Theorem 2.2 Let $\mathbb{X}_{i}, i \in I$, be pairwise disjoint and connected $L_{b}$-structures. Then the structure $\bigcup_{i \in I} \mathbb{X}_{i}$ is reversible iff whenever $f: I \rightarrow I$ is a surjection, $g_{i} \in \operatorname{Mono}\left(\mathbb{X}_{i}, \mathbb{X}_{f(i)}\right)$, for $i \in I$, and

$$
\forall j \in I \quad\left(\left\{g_{i}\left[X_{i}\right]: i \in f^{-1}[\{j\}]\right\} \text { is a partition of } X_{j}\right)
$$

we have

$$
f \in \operatorname{Sym}(I) \wedge \forall i \in I \quad g_{i} \in \operatorname{Iso}\left(\mathbb{X}_{i}, \mathbb{X}_{f(i)}\right)
$$

Proof. Let $\mathbb{X}_{i}=\left\langle X_{i}, \rho_{i}\right\rangle$, for $i \in I$, and let $\mathbb{X}=\langle X, \rho\rangle=\left\langle\bigcup_{i \in I} X_{i}, \bigcup_{i \in I} \rho_{i}\right\rangle$.

Suppose that $\mathbb{X}$ is a reversible structure and let the mappings $f$ and $g_{i}$ be as assumed. Then by Fact 1.5 (a) we have $F:=\bigcup_{i \in I} g_{i} \in \operatorname{Cond}(\mathbb{X})=\operatorname{Aut}(\mathbb{X})$ and, by Fact 1.5 (b), $g_{i} \in \operatorname{Emb}\left(\mathbb{X}_{i}, \mathbb{X}_{f(i)}\right)$, for all $i \in I$. Suppose that there are different $i_{1}, i_{2} \in I$ such that $f\left(i_{1}\right)=f\left(i_{2}\right)=j$ and let $x_{1} \in X_{i_{1}}$ and $x_{2} \in$ $X_{i_{2}}$. Since the structure $\mathbb{X}_{j}$ is connected there are $y_{1}, \ldots, y_{n} \in X_{j}$ such that $g_{i_{1}}\left(x_{1}\right)=y_{1}\left(\rho_{j}\right)_{s} y_{2} \ldots\left(\rho_{j}\right)_{s} y_{n}=g_{i_{2}}\left(x_{2}\right)$ and, hence, there is $k<n$ such that $y_{k}$ and $y_{k+1}$ are in different elements of the partition $\left\{g_{i}\left[X_{i}\right]: i \in I \wedge f(i)=j\right\}$, say $y_{k}=g_{i}(x) \in g_{i}\left[X_{i}\right]$ and $y_{k+1}=g_{i^{\prime}}\left(x^{\prime}\right) \in g_{i^{\prime}}\left[X_{i^{\prime}}\right]$, where $i \neq i^{\prime}$. But then $\left\langle g_{i}(x), g_{i^{\prime}}\left(x^{\prime}\right)\right\rangle \in \rho_{r s}$, which is, by Fact 1.5 (b), impossible. Thus $f$ is a bijection and, by (4), for each $i \in I$ we have $g_{i}\left[X_{i}\right]=X_{f(i)}$ and, hence, $g_{i} \in \operatorname{Iso}\left(\mathbb{X}_{i}, \mathbb{X}_{f(i)}\right)$.

Conversely, for $F \in \operatorname{Cond}(\mathbb{X})$ we prove that $F \in \operatorname{Aut}(\mathbb{X})$. By Fact 1.5 (a) and the assumption we have $F=\bigcup_{i \in I} g_{i}$, where $f \in \operatorname{Sym}(I)$ and $g_{i} \in \operatorname{Iso}\left(\mathbb{X}_{i}, \mathbb{X}_{f(i)}\right)$, for all $i \in I$. By Fact 1.5 (b) we have $F \in \operatorname{Emb}(\mathbb{X})$ and, since $F$ is a surjection, $F \in \operatorname{Aut}(\mathbb{X})$.

Now we prove Theorem 1.2. In fact we will prove its contrapositive.

Theorem $2.3(*)$ The union $\mathbb{X}:=\bigcup_{i \in I} \mathbb{X}_{i}$ is not reversible iff $\left\langle\mathbb{X}_{i}: i \in I\right\rangle$ is not a reversible sequence of structures or there is a non-trivial $\mathbb{Z}$-sequence in $I$. 
Proof. $(\Rightarrow)$ If $\mathbb{X}$ is not a reversible structure, then by Theorem 2.2 there are $f \in$ $\operatorname{Sur}(I)$ and $g_{i} \in \operatorname{Mono}\left(\mathbb{X}_{i}, \mathbb{X}_{f(i)}\right)$, for $i \in I$, satisfying (4) and $\neg(5)$. By (4) and Fact 1.5 (a) we have $\bigcup_{i \in f^{-1}[\{j\}]} g_{i} \in \operatorname{Cond}\left(\bigcup_{i \in f^{-1}[\{j\}]} \mathbb{X}_{i}, \mathbb{X}_{j}\right)$, for all $j \in I$. So, if $f \notin \operatorname{Sym}(I)$, then we have $\neg(2)$.

If $f \in \operatorname{Sym}(I)$, then, by $\neg(5), g_{i_{0}} \notin \operatorname{Iso}\left(\mathbb{X}_{i_{0}}, \mathbb{X}_{f\left(i_{0}\right)}\right)$, for some $i_{0} \in I$. Since $g_{i_{0}} \in \operatorname{Cond}\left(\mathbb{X}_{i_{0}}, \mathbb{X}_{f\left(i_{0}\right)}\right)$ and, since by the reversibility of $\mathbb{X}_{i_{0}}$ and Fact $1.4, \mathbb{X}_{i_{0}} \cong$ $\mathbb{X}_{f\left(i_{0}\right)}$ would imply Cond $\left(\mathbb{X}_{i_{0}}, \mathbb{X}_{f\left(i_{0}\right)}\right)=\operatorname{Iso}\left(\mathbb{X}_{i_{0}}, \mathbb{X}_{f\left(i_{0}\right)}\right)$, we have $\mathbb{X}_{i_{0}} \not \mathbb{X}_{f\left(i_{0}\right)}$.

Let $i_{k}:=f^{k}\left(i_{0}\right)$, for $k \in \mathbb{Z}$. Then $\mathbb{X}_{i_{0}} ¥ \mathbb{X}_{i_{1}}$ and for each $k \in \mathbb{Z}$ we have $f^{-1}\left[\left\{i_{k+1}\right\}\right]=\left\{i_{k}\right\}$ and, hence, $g_{i_{k}} \in \operatorname{Cond}\left(\mathbb{X}_{i_{k}}, \mathbb{X}_{i_{k+1}}\right)$.

Suppose $i_{k}=i_{l}$, for some $k<l \in \mathbb{Z}$. Then $f^{l}\left(i_{0}\right)=f^{k}\left(i_{0}\right)$, and, hence, $i_{l-k}=f^{l-k}\left(i_{0}\right)=i_{0}$, where $l-k \geq 1$. So, $\mathbb{X}_{i_{0}} \preccurlyeq c \mathbb{X}_{i_{1}} \preccurlyeq c \cdots \preccurlyeq_{c} \mathbb{X}_{i_{l-k}}=\mathbb{X}_{i_{0}}$, which implies $\mathbb{X}_{i_{0}} \sim_{c} \mathbb{X}_{i_{1}}$. Since $\mathbb{X}_{i_{0}}$ is a reversible structure, by Fact 1.4 we would have $\mathbb{X}_{i_{0}} \cong \mathbb{X}_{i_{1}}$, which is false. So $\left\langle i_{k}: k \in \mathbb{Z}\right\rangle$ is an injection and, since $\mathbb{X}_{i_{0}} ¥ \mathbb{X}_{i_{1}}$, it is a non-trivial $\mathbb{Z}$-sequence in $I$.

$(\Leftarrow)$ If there are $f \in \operatorname{Sur}(I) \backslash \operatorname{Sym}(I)$ and $G_{j} \in \operatorname{Cond}\left(\bigcup_{i \in f^{-1}[\{j\}]} \mathbb{X}_{i}, \mathbb{X}_{j}\right)$, for $j \in I$, then, clearly, $g_{i}:=G_{f(i)} \uparrow X_{i} \in \operatorname{Mono}\left(\mathbb{X}_{i}, \mathbb{X}_{f(i)}\right)$, for all $i \in I$, and (4) is true. Since $f \notin \operatorname{Sym}(I)$, by Theorem 2.2 the structure $\mathbb{X}$ is not reversible.

Suppose that $\left\langle i_{k}: k \in \mathbb{Z}\right\rangle$ is a $\mathbb{Z}$-sequence in $I$ and that $\mathbb{X}_{i_{r}} ¥ \mathbb{X}_{i_{r+1}}$, for some $r \in \mathbb{Z}$. Then the function $f: I \rightarrow I$, defined by $f(i)=i$, for $i \in I \backslash\left\{i_{k}: k \in \mathbb{Z}\right\}$, and $f\left(i_{k}\right)=i_{k+1}$, for $k \in \mathbb{Z}$, is a bijection. Let $g_{i}:=\operatorname{id}_{X_{i}}$, for $i \in I \backslash\left\{i_{k}: k \in \mathbb{Z}\right\}$, and let us take $g_{i_{k}} \in \operatorname{Cond}\left(\mathbb{X}_{i_{k}}, \mathbb{X}_{i_{k+1}}\right)$, for $k \in \mathbb{Z}$. Then $g_{i} \in \operatorname{Mono}\left(\mathbb{X}_{i}, \mathbb{X}_{f(i)}\right)$, for all $i \in I$, and (4) holds. But $g_{i_{r}} \notin \operatorname{Iso}\left(\mathbb{X}_{i_{r}}, \mathbb{X}_{i_{r+1}}\right)$ and, by Theorem 2.2, the structure $\mathbb{X}$ is not reversible.

Corollary 2.4 An $L_{b}$-structure with finitely many components is reversible iff all its components are reversible.

Proof. Let $\mathbb{X}=\bigcup_{i \in I} \mathbb{X}_{i}$, where $|I|<\omega$ and $\mathbb{X}_{i}, i \in I$, are pairwise disjoint and connected $L_{b}$-structures. The implication " $\Rightarrow$ " follows from Theorem 2.1. If the structures $\mathbb{X}_{i}, i \in I$, are reversible, then $(*)$ holds, so, since $\operatorname{Sur}(I)=\operatorname{Sym}(I)$ and there are no $\mathbb{Z}$-sequences in $I$, by Theorem 2.3 the structure $\mathbb{X}$ is reversible.

Corollary 2.5 An $L_{b}$-structure $\bigcup_{i \in I} \mathbb{X}_{i}$ with finite components is reversible iff $\left\langle\mathbb{X}_{i}: i \in I\right\rangle$ is a reversible sequence of structures and there are no infinite classes $\left[\mathbb{X}_{i}\right] \cong,\left[\mathbb{X}_{j}\right] \cong \in \mathcal{X} / \cong$ such that $\mathbb{X}_{i} \prec_{c} \mathbb{X}_{j}$.

Proof. Since all finite structures are reversible condition $(*)$ is fulfilled. According to Theorem 1.2 we show that the negation of the second condition holds iff there is a non-trivial $\mathbb{Z}$-sequence in $I$. The implication “ $\Rightarrow$ ” is trivial. 
If $\left\langle i_{k}: k \in \mathbb{Z}\right\rangle$ is a non-trivial $\mathbb{Z}$-sequence in $I$, then $\mathbb{X}_{i_{k}} \preccurlyeq c \mathbb{X}_{i_{k+1}}$, for all $k \in$ $\mathbb{Z}$, and there is $k_{0} \in \mathbb{Z}$ such that $\mathbb{X}_{i_{k_{0}}} \prec_{c} \mathbb{X}_{i_{k_{0}+1}}$. Since $\mathbb{X}_{i_{k_{0}+1}} \preccurlyeq_{c} \mathbb{X}_{i_{k_{0}+2}} \preccurlyeq c \ldots$ and the structures $\mathbb{X}_{i}, i \in I$, are finite, there is $s \geq k_{0}+1$ such that $\mathbb{X}_{i_{k}} \cong \mathbb{X}_{i_{s}}$, for all $k \geq s$, and, similarly, there is $r \leq k_{0}$ such that $\mathbb{X}_{i_{k}} \cong \mathbb{X}_{i_{r}}$, for all $k \leq r$. Now we have $\mathbb{X}_{i_{r}} \prec \mathbb{X}_{i_{s}}$ and the classes $\left[\mathbb{X}_{i_{r}}\right] \cong$ and $\left[\mathbb{X}_{i_{s}}\right] \cong$ are infinite.

By Theorem 3.4 of [10], if $\mathbb{X}_{i}, i \in I$, are pairwise disjoint tournaments (resp. in particular, linear orders), and $\left\langle\left|X_{i}\right|: i \in I\right\rangle$ is a reversible sequence of cardinals, then the digraph (resp. poset) $\bigcup_{i \in I} \mathbb{X}_{i}$ is reversible. But that condition is not necessary for the reversibility of such unions and now we give a characterization.

Corollary 2.6 A disjoint union $\bigcup_{i \in I} \mathbb{X}_{i}$ of linear orders (or, more generally, tournaments) is not reversible iff there is a non-injective surjection $f: I \rightarrow I$ such that each component $\mathbb{X}_{j}$ can be partitioned into copies of $\mathbb{X}_{i}$, where $i \in f^{-1}[\{j\}] .6$

Proof. $(*)$ is true, because all tournaments are reversible and connected. Since for any two tournaments $\mathbb{X}$ and $\mathbb{Y}$ we have $\operatorname{Cond}(\mathbb{X}, \mathbb{Y})=\operatorname{Iso}(\mathbb{X}, \mathbb{Y})$, all $\mathbb{Z}$-sequences in $I$ are trivial and, by Theorem 2.3, $\bigcup_{i \in I} \mathbb{X}_{i}$ is not reversible iff there is $f \in$ $\operatorname{Sur}(I) \backslash \operatorname{Sym}(I)$ such that for each $j \in I$ there is $G_{j} \in \operatorname{Cond}\left(\bigcup_{i \in f^{-1}[\{j\}]} \mathbb{X}_{i}, \mathbb{X}_{j}\right)$.

Then for $i \in f^{-1}[\{j\}]$ and $A_{i}:=G_{j}\left[X_{i}\right]$ we have $G_{j}\left\lceil X_{i} \in \operatorname{Cond}\left(\mathbb{X}_{i}, \mathbb{A}_{i}\right)=\right.$ $\operatorname{Iso}\left(\mathbb{X}_{i}, \mathbb{A}_{i}\right)$ and $\left\{A_{i}: i \in f^{-1}[\{j\}]\right\}$ is a partition of $X_{j}$.

Conversely, if $f \in \operatorname{Sur}(I) \backslash \operatorname{Sym}(I)$ and $\left\{A_{i}: i \in f^{-1}[\{j\}]\right\}$ is a partition of $X_{j}$, for each $j \in I$, and $g_{i} \in \operatorname{Iso}\left(\mathbb{X}_{i}, \mathbb{A}_{i}\right)$, for $i \in f^{-1}[\{j\}]$, then, clearly, $G_{j}:=\bigcup_{\left.i \in f^{-1}[\{j\}]\right\}} g_{i} \in \operatorname{Cond}\left(\bigcup_{i \in f^{-1}[\{j\}]} \mathbb{X}_{i}, \mathbb{X}_{j}\right)$ and we are done.

\section{Triviality of $\omega^{*}$-sequences of monomorphisms}

In this and the following section we consider some conditions which imply reversibility of disconnected $L_{\beta}$-structures. If $\mathbb{X}$ and $\mathbb{Y}$ are $L_{b}$-structures and there is a monomorphism $f: \mathbb{X} \rightarrow \mathbb{Y}$, we will write $\mathbb{X} \preccurlyeq m \mathbb{Y}$.

Under (*), a mapping $i: \omega \rightarrow I$, usually denoted by $\left\langle i_{k}: k \in \omega\right\rangle$, will be called an $\omega^{*}$-sequence in $I$ iff it is an injection and

$$
\forall k, l \in \omega\left(k<l \Rightarrow \mathbb{X}_{i_{l}} \preccurlyeq m \mathbb{X}_{i_{k}}\right) .
$$

If, in addition, $\operatorname{Mono}\left(\mathbb{X}_{i_{1}}, \mathbb{X}_{i_{0}}\right)=\operatorname{Iso}\left(\mathbb{X}_{i_{1}}, \mathbb{X}_{i_{0}}\right)$, the $\omega^{*}$-sequence $\left\langle i_{k}: k \in \omega\right\rangle$ will be called trivial. By the transitivity of the relation $\preccurlyeq_{m}$, condition (6) is equivalent to the existence of a sequence of monomorphisms $g_{k}: \mathbb{X}_{i_{k+1}} \rightarrow \mathbb{X}_{i_{k}}, k \in \omega 7$

\footnotetext{
${ }^{6}$ that is, there is a partition $\left\{A_{i}: i \in f^{-1}[\{j\}]\right\}$ of $X_{j}$ such that $\mathbb{A}_{i} \cong \mathbb{X}_{i}$, for all $i \in f^{-1}[\{j\}]$.

${ }^{7}$ We note that $\left\langle i_{k}: k \in \omega\right\rangle$ is an $\omega^{*}$-sequence in $I$ iff $k \mapsto \mathbb{X}_{i_{k}}$ is a monomorphism from the linear order $\left.\omega^{*}=\langle\omega\rangle,\right\rangle$ to the preorder $\langle\mathcal{X}, \preccurlyeq m\rangle$ and $\left\langle i_{k}: k \in \omega\right\rangle$ is non-trivial iff we can choose $g_{0}: \mathbb{X}_{i_{1}} \rightarrow \mathbb{X}_{i_{0}}$ which is not an isomorphism. This holds if, in particular, $\mathbb{X}_{i_{1}} \neq \mathbb{X}_{i_{0}}$.
} 
Fact 3.1 Let $f: I \rightarrow I$ be a surjection, let $j \in I$, where $\mid f^{-1}[\{j\} \mid>1$, and let $O(j):=\left\{f^{n}(j): n \in \omega\right\}$. Then $\left|f^{-1}[\{j\}] \cap O(j)\right| \leq 1$.

Proof. If there exists $i \in f^{-1}[\{j\}] \cap O(j)$, then $i=f^{k}(j)$, for some $k \in \omega$, and $j=f(i)=f^{k+1}(j)$. So, there is $l=\min \left\{m \in \omega: f^{m}(j)=j\right\}$.

If $l=0$, that is $f(j)=j$, then $O(j)=\{j\}$ and the statement is true.

If $l>0$, then $f\left(f^{l-1}(j)\right)=f^{l}(j)=j$ and, hence, $f^{l-1}(j) \in f^{-1}[\{j\}] \cap O(j)$. Clearly, $f^{q l}(j)=j$, for all $q \in \omega$. So, if $n=q l+r$, where $q \in \omega$ and $r<l$, then $f^{n}(j)=f^{r}\left(f^{q l}(j)\right)=f^{r}(j)$ and, hence, $O(j)=\left\{f^{n}(j): n \leq l-1\right\}$. Assuming that $f^{n}(j) \in f^{-1}[\{j\}] \cap O(j)$, for some $n<l-1$, we would have $f^{n+1}(j)=j$ and $n+1<l$, which contradicts the minimality of $l$. Thus $f^{-1}[\{j\}] \cap O(j)=$ $\left\{f^{l-1}(j)\right\}$ and we are done.

Theorem $3.2(*)$ If each $\omega^{*}$-sequence in I is trivial, 8 the structure $\mathbb{X}$ is reversible.

Proof. If $\mathbb{X}$ is not reversible, then by Theorem 2.3 we have the following two cases.

Case 1. There is a $\mathbb{Z}$-sequence $\left\langle i_{k}: k \in \mathbb{Z}\right\rangle$ in $I$ such that $\mathbb{X}_{i_{-1}} \neq \mathbb{X}_{i_{0}}$. Let $j_{k}:=i_{-k}$, for $k \in \mathbb{Z}$. Then for each $k \in \omega$ we have $\operatorname{Mono}\left(\mathbb{X}_{j_{k+1}}, \mathbb{X}_{j_{k}}\right) \supset$ $\operatorname{Cond}\left(\mathbb{X}_{i_{-(k+1)}}, \mathbb{X}_{i_{-k}}\right) \neq \emptyset$ and, hence, $\left\langle j_{k}: k \in \omega\right\rangle$ is an $\omega^{*}$-sequence in $I$ and $\operatorname{Mono}\left(\mathbb{X}_{j_{1}}, \mathbb{X}_{j_{0}}\right) \neq \operatorname{Iso}\left(\mathbb{X}_{j_{1}}, \mathbb{X}_{j_{0}}\right)=\emptyset$.

Case 2. There is $f \in \operatorname{Sur}(I) \backslash \operatorname{Sym}(I)$ such that for each $j \in I$ there exists $G_{j} \in \operatorname{Cond}\left(\bigcup_{i \in f^{-1}[\{j\}]} \mathbb{X}_{i}, \mathbb{X}_{j}\right)$. Let $j_{*} \in I$, where $\mid f^{-1}\left[\left\{j_{*}\right\} \mid>1\right.$. By Fact 3.1 there is

$$
i_{*} \in f^{-1}\left[\left\{j_{*}\right\}\right] \backslash\left\{f^{n}\left(j_{*}\right): n \in \omega\right\} .
$$

Since $f: I \rightarrow I$ is a surjection, there is a sequence $\left\langle i_{k}: k \in \omega\right\rangle \in I^{\omega}$, such that $i_{0}=j_{*}, i_{1}=i_{*}$, and $f\left(i_{k+1}\right)=i_{k}$, for all $k \in \omega$. Suppose that there is $k \in \mathbb{N}$ such that $i_{k} \in\left\{f^{n}\left(j_{*}\right): n \in \omega\right\}$ and let $k$ be the minimal such element of $\mathbb{N}$. By (7) we have $k>1$. So $i_{k}=f^{n}\left(j_{*}\right)$, for some $n \in \omega$, and, hence, $i_{k-1}=f\left(i_{k}\right)=f^{n+1}\left(j_{*}\right)$, which contradicts the minimality of $k$. Thus

$$
\left\{i_{k}: k \in \mathbb{N}\right\} \cap\left\{f^{n}\left(j_{*}\right): n \in \omega\right\}=\emptyset .
$$

Suppose that $i: \omega \rightarrow I$ is not an injection and let $r$ be the minimal element of $\omega$ such that $i_{r}=i_{s}$, for some $s>r . r=0$ would imply that $i_{s}=i_{0}=j_{*} \in$ $\left\{f^{n}\left(j_{*}\right): n \in \omega\right\}$, which is impossible by (8). Now $i_{r-1}=f\left(i_{r}\right)=f\left(i_{s}\right)=i_{s-1}$, which is impossible by the minimality of $r$. Thus $i: \omega \rightarrow I$ is an injection.

For $k \in \omega$ we have $G_{i_{k}} \in \operatorname{Cond}\left(\bigcup_{i \in f^{-1}\left[\left\{i_{k}\right\}\right]} \mathbb{X}_{i}, \mathbb{X}_{i_{k}}\right)$ and $i_{k+1} \in f^{-1}\left[\left\{i_{k}\right\}\right]$ so $G_{i_{k}}\left\lceil X_{i_{k+1}} \in \operatorname{Mono}\left(\mathbb{X}_{i_{k+1}}, \mathbb{X}_{i_{k}}\right)\right.$. Since $\left|f^{-1}\left[\left\{i_{0}\right\}\right]\right|>1$, is follows that

\footnotetext{
${ }^{8}$ Then each monomorphism $i: \omega^{*} \rightarrow\langle\mathcal{X}, \preccurlyeq m\rangle$ maps $\omega$ into the $\cong$-class of $\mathbb{X}_{i_{0}}$ (if $\mathbb{X}_{i_{0}} ¥ \mathbb{X}_{i_{k}}$, then $\left\langle i_{0}, i_{k}, i_{k+1}, \ldots\right\rangle$ is a non-trivial $\omega^{*}$-sequence in $I$ ) but the converse is not true (take $\bigcup_{\omega} \omega$ ).
} 
$G_{i_{0}}\left\lceil X_{i_{1}} \in \operatorname{Mono}\left(\mathbb{X}_{i_{1}}, \mathbb{X}_{i_{0}}\right) \backslash \operatorname{Iso}\left(\mathbb{X}_{i_{1}}, \mathbb{X}_{i_{0}}\right)\right.$. Therefore, $\left\langle i_{k}: k \in \omega\right\rangle$ is a non-trivial $\omega^{*}$-sequence in $I$.

Example 3.3 The converse of Theorem 3.2 is not true. The equivalence relation $\bigcup_{i \in \omega} \mathbb{X}_{i}$, where $\left|X_{i}\right|=2$, for even $i$ 's, and $\left|X_{i}\right|=5$, for odd $i$ 's, is reversible [10] (see, also, Introduction) and $\langle 1,0,2,4,6, \ldots\rangle$ is a non-trivial $\omega^{*}$-sequence in $\omega$.

Remark 3.4 If each $\omega^{*}$-sequence in $I$ is trivial, then we have two possibilities:

1. There is an $\omega^{*}$-sequence $\left\langle i_{k}: k \in \omega\right\rangle$. Then there is $g_{0} \in \operatorname{Mono}\left(\mathbb{X}_{i_{1}}, \mathbb{X}_{i_{0}}\right)=$ Iso $\left(\mathbb{X}_{i_{1}}, \mathbb{X}_{i_{0}}\right)$, which implies that $\mathbb{X}_{i_{1}} \cong \mathbb{X}_{i_{0}}$ and $\operatorname{Mono}\left(\mathbb{X}_{i_{0}}\right)=\operatorname{Aut}\left(\mathbb{X}_{i_{0}}\right)$. Generally speaking, if $\mathbb{X}$ is a structure satisfying $\operatorname{Mono}(\mathbb{X})=\operatorname{Aut}(\mathbb{X})$, then $\operatorname{Cond}(\mathbb{X})=$ $\operatorname{Emb}(\mathbb{X})=\operatorname{Aut}(\mathbb{X})$; so it is reversible and copy-minimal (see [7] for examples). Clearly, if $\mathbb{X}$ is a finite structure, then $\operatorname{Mono}(\mathbb{X})=\operatorname{Aut}(\mathbb{X})$ and the linear graph $\mathbb{G}_{\mathbb{Z}}$ is a reversible, connected infinite structure satisfying $\operatorname{Mono}(\mathbb{X})=\operatorname{Aut}(\mathbb{X})$. We note that in [1] Dushnik and Miller constructed embedding-rigid dense suborders $\mathbb{L}$ of the real line (i.e. $\operatorname{Emb}(\mathbb{L})=\left\{\mathrm{id}_{L}\right\}$; see also [17], p. 147) of size $\mathfrak{c}$ and similar examples can be made using the ZFC result of Vopěnka, Pultr and Hedrlín [19] saying that on every set there is an endo-rigid binary relation.

2. $\omega^{*}$-sequences do not exist at all. This situation is considered in the sequel.

\section{Non-existence of $\omega^{*}$-sequences. Monotone functions}

We recall that a pair $\mathcal{W}=\langle\mathcal{A}, \mathcal{R}\rangle$ is called a well founded relation (we will write $\mathcal{W} \in$ Wfr) iff $\mathcal{A}$ is a class, $\mathcal{R}$ a binary class relation on $\mathcal{A}$ and each non-empty set $X \subset \mathcal{A}$ has an $\mathcal{R}$-minimal element, that is,

$$
\forall X \quad(\emptyset \neq X \subset \mathcal{A} \Rightarrow \exists y \in X \neg \exists z \in X \quad z \mathcal{R} y) \emptyset
$$

Note that then the relation $\mathcal{R}$ on $\mathcal{A}$ is irreflexive and asymmetric (a "class-digraph") and its reflexivization $\leq_{\mathcal{R}}$ is defined by

$$
a \leq_{\mathcal{R}} b \Leftrightarrow a=b \vee a \mathcal{R} b .
$$

If, in addition, $\mathcal{C} \subset \operatorname{Mod}_{L}$ is a class of $L$-structures, we will say that a (class) function $\theta: \mathcal{C} \rightarrow \mathcal{A}$ is monotone with respect to monomorphisms iff

$$
\forall \mathbb{X}, \mathbb{Y} \in \mathcal{C}\left(\mathbb{X} \preccurlyeq_{m} \mathbb{Y} \Rightarrow \theta(\mathbb{X}) \leq_{\mathcal{R}} \theta(\mathbb{Y})\right)
$$

\footnotetext{
${ }^{9}$ For convenience, we use that general notions from set theory. The classes $\mathcal{A}$ and $\mathcal{R}$ are collections of sets satisfying some formulas, say $A(v)$ and $R(u, v)$ of the language of set theory, $\{\in\}$. For example, $\mathcal{A}$ is the class of all ordinals and $\mathcal{R}$ the usual strict linear order on that class.
} 
and the class of such functions (which are, in fact, homomorphisms from the preorder $\left\langle\mathcal{C}, \preccurlyeq_{m}\right\rangle$ to the reflexivization of $\left.\mathcal{W}\right)$ will be denoted by $\mathcal{M}(\mathcal{C}, \mathcal{W})$.

If $(*)$ holds, let $\mathcal{M}(\mathcal{X})=\bigcup_{\mathcal{W} \in \mathrm{Wfr}} \mathcal{M}(\mathcal{X}, \mathcal{W})$. For $\theta \in \mathcal{M}(\mathcal{X}, \mathcal{W})$ and $a \in \mathcal{A}$, let

$$
I_{a}^{\theta}:=\left\{i \in I: \theta\left(\mathbb{X}_{i}\right)=a\right\}
$$

Theorem 4.1 (*) If there is $\theta \in \mathcal{M}(\mathcal{X})$ such that for each $a \in \theta[\mathcal{X}]$ there are no $\omega^{*}$-sequences in $I_{a}^{\theta}$, then $\mathbb{X}$ is a reversible structure. This holds if, in particular, $\left\langle\theta\left(\mathbb{X}_{i}\right): i \in I\right\rangle$ is a finite-to-one sequence (i.e., if the sets $I_{a}^{\theta}, a \in \theta[\mathcal{X}]$, are finite).

Proof. First we prove that the following conditions are equivalent:

(i) There is an $\omega^{*}$-sequence in $I$,

(ii) $\forall \theta \in \mathcal{M}(\mathcal{X}) \quad \exists a \in \theta[\mathcal{X}] \quad \exists \omega^{*}$-sequence in $I_{a}^{\theta}$,

(iii) $\exists \theta \in \mathcal{M}(\mathcal{X}) \exists a \in \theta[\mathcal{X}] \exists \omega^{*}$-sequence in $I_{a}^{\theta}$.

(i) $\Rightarrow$ (ii). Let $\left\langle i_{k}: k \in \omega\right\rangle$ be an $\omega^{*}$-sequence in $I, \mathcal{W} \in \mathrm{Wfr}$ and $\theta \in \mathcal{M}(\mathcal{X}, \mathcal{W})$. Since $X=\left\{\theta\left(\mathbb{X}_{i_{k}}\right): k \in \omega\right\}$ is a non-empty subset of $\mathcal{A}$, by (9) there is $k_{0} \in \omega$ such that

$$
\forall k \in \omega \neg \theta\left(\mathbb{X}_{i_{k}}\right) \mathcal{R} \theta\left(\mathbb{X}_{i_{k_{0}}}\right) .
$$

Now for $k \geq k_{0}$ we have $\mathbb{X}_{i_{k}} \preccurlyeq m \mathbb{X}_{i_{0}}$, which by (10) implies $\theta\left(\mathbb{X}_{i_{k}}\right) \leq_{\mathcal{R}}$ $\theta\left(\mathbb{X}_{i_{k_{0}}}\right)=: a$ and, by $(12), \theta\left(\mathbb{X}_{i_{k}}\right)=\theta\left(\mathbb{X}_{i_{k_{0}}}\right)$. So $\theta\left(\mathbb{X}_{i_{k}}\right)=a$, for all $k \geq k_{0}$, that is $\left\{i_{k}: k \geq k_{0}\right\} \subset I_{a}^{\theta}$ and $\left\langle i_{k_{0}+k}: k \in \omega\right\rangle$ is an $\omega^{*}$-sequence in $I_{a}^{\theta}$.

(ii) $\Rightarrow$ (iii). If Card denotes the class of all cardinals, then, clearly, $\mathcal{W}=$ $\langle$ Card,$<\rangle \in \mathrm{Wfr}$ and for the function $\theta: \mathcal{X} \rightarrow$ Card defined by $\theta\left(\mathbb{X}_{i}\right)=\left|X_{i}\right|$ we have $\theta \in \mathcal{M}(\mathcal{X}, \mathcal{W})$. By (ii) there are a cardinal $\kappa$ and an $\omega^{*}$-sequence in $I_{\kappa}^{\theta}$.

(iii) $\Rightarrow$ (i). This is trivial, since an $\omega^{*}$-sequence in $I_{a}^{\theta}$ is an $\omega^{*}$-sequence in $I$. Now, by the assumption, $\neg$ (ii) holds and, hence, there are no $\omega^{*}$-sequences in $I$ and we apply Theorem 3.2 .

Corollary 4.2 If $(*)$ holds and the sequence $\left\langle\left|X_{i}\right|: i \in I\right\rangle$ is finite-to-one, 10 then $\bigcup_{i \in I} \mathbb{X}_{i}$ is a reversible structure.

For the structures with finite components the condition implying the reversibility of $\bigcup_{i \in I} \mathbb{X}_{i}$ given in Theorem 4.1 is, in fact, equivalent to a simpler condition.

Proposition 4.3 If $(*)$ holds and the structures $\mathbb{X}_{i}, i \in I$, are finite, then the following conditions are equivalent:

(a) The sequence $\left\langle\left|X_{i}\right|: i \in I\right\rangle$ is finite-to-one,

(b) $\exists \theta \in \mathcal{M}(\mathcal{X}) \forall a \in \theta[\mathcal{X}] \neg \exists \omega^{*}$-sequence in $I_{a}^{\theta}$.

\footnotetext{
${ }^{10}$ that is, there is no infinite $J \subset I$ such that $\left|\mathbb{X}_{i}\right|=\left|\mathbb{X}_{j}\right|$, for all $i, j \in J$.
} 
Proof. (a) $\Rightarrow$ (b) is trivial: take $\theta: \mathcal{X} \rightarrow$ Card, where $\theta\left(\mathbb{X}_{i}\right)=\left|X_{i}\right|$.

$\neg$ (a) $\Rightarrow \neg$ (b). Let $J \subset I$ and $n \in \mathbb{N}$, where $|J| \geq \omega$ and $\left|X_{i}\right|=n$, for all $i \in J$. Then, since the structures $\mathbb{X}_{i}, i \in I$, are finite, there is $K \subset J$, where $|K| \geq \omega$ and $\mathbb{X}_{i} \cong \mathbb{X}_{j}$, for all $i, j \in K$; let us fix an $i_{0} \in K$. Let $\theta \in \mathcal{M}(\mathcal{X}, \mathcal{W})$ and $a \in \theta[\mathcal{X}]$, where $\theta\left(\mathbb{X}_{i_{0}}\right)=a$. Now, if $i \in K$, then $\mathbb{X}_{i} \cong \mathbb{X}_{i_{0}}$ and, hence $\mathbb{X}_{i} \preccurlyeq_{m} \mathbb{X}_{i_{0}} \preccurlyeq_{m} \mathbb{X}_{i}$, which implies that $\theta\left(\mathbb{X}_{i}\right) \leq_{\mathcal{R}} \theta\left(\mathbb{X}_{i_{0}}\right) \leq_{\mathcal{R}} \theta\left(\mathbb{X}_{i}\right)$, and, thus, $\theta\left(\mathbb{X}_{i}\right)=a$, that is $i \in I_{a}^{\theta}$. So $K \subset I_{a}^{\theta}$ and taking an injection $i: \omega \rightarrow K$ we obtain an $\omega^{*}$-sequence $\left\langle i_{k}: k \in \omega\right\rangle$ in $I_{a}^{\theta}$ (because $\mathbb{X}_{i_{k+1}} \cong \mathbb{X}_{i_{k}}$ gives $\mathbb{X}_{i_{k+1}} \preccurlyeq m \mathbb{X}_{i_{k}}$ ).

Finite diagonal products of monotone functions The class Wfr is not closed under direct products (in the product of two-element chains, $2^{\omega}$, the set $X=\left\{x_{n}\right.$ : $n \in \omega\}$, where $x_{n}=\langle 0, \ldots, 0,1,1, \ldots\rangle$ has $n$-many zeros, does not have a minimal element). But Wfr is closed under finite products.

Theorem 4.4 Let $n \in \mathbb{N}$ and let $\mathcal{W}_{k}=\left\langle\mathcal{A}_{k}, \mathcal{R}_{k}\right\rangle \in \mathrm{Wfr}$, for $k<n$. Then

(a) $\left\langle\prod_{k<n} \mathcal{A}_{k}, \mathcal{R}\right\rangle \in \mathrm{Wfr}$, where for $a=\left\langle a_{k}\right\rangle, b=\left\langle b_{k}\right\rangle \in \prod_{k<n} \mathcal{A}_{k}$ we have

$$
a \mathcal{R} b \Leftrightarrow \forall k<n\left(a_{k}=b_{k} \vee a_{k} \mathcal{R}_{k} b_{k}\right) \wedge \exists k<n a_{k} \mathcal{R}_{k} b_{k} ;
$$

(b) If $\mathcal{C} \subset \operatorname{Mod}_{L}$ and $\theta_{k} \in \mathcal{M}\left(\mathcal{C}, \mathcal{W}_{k}\right)$, for $k<n$, then $\theta \in \mathcal{M}\left(\mathcal{C}, \prod_{k<n} \mathcal{A}_{k}\right)$, where $\theta$ is the diagonal mapping $\theta=\Delta_{k<n} \theta_{k}: \mathcal{C} \rightarrow \prod_{k<n} \mathcal{A}_{k}$, defined by

$$
\theta(\mathbb{X})=\left\langle\theta_{k}(\mathbb{X}): k<n\right\rangle ;
$$

(c) If (*) holds and in (b) we put $\mathcal{C}=\mathcal{X}$, then for $a=\left\langle a_{k}: k<n\right\rangle \in \theta[\mathcal{X}]$ we have

$$
I_{a}^{\theta}=\left\{i \in I: \forall k<n \quad \theta_{k}(\mathbb{X})=a_{k}\right\}=\bigcap_{k<n} I_{a_{k}}^{\theta_{k}}
$$

Thus the partition $\left\{I_{a}^{\theta}: a \in \theta[\mathcal{X}]\right\}$ of I refines all partitions $\left\{I_{a_{k}}^{\theta_{k}}: a_{k} \in \theta_{k}[\mathcal{X}]\right\}$, $k<n$, and, hence, the sequence $\left\langle\theta\left(\mathbb{X}_{i}\right): i \in I\right\rangle$ has more chance to be finite-toone (see Theorem 4.1).

Proof. (a) Suppose that a non-empty set $X \subset \prod_{k<n} \mathcal{A}_{k}$ has no $\mathcal{R}$-minimal elements. Then there are $a^{r} \in X, r \in \omega$, such that for each $r \in \omega$ we have $a^{r+1} \mathcal{R} a^{r}$ and, by (13), there is $k_{r}<n$ satisfying $a_{k_{r}}^{r+1} \mathcal{R}_{k_{r}} a_{k_{r}}^{r}$. Thus there are $k^{*}<n$ and an increasing sequence $\left\langle r_{s}: s \in \omega\right\rangle$ in $\omega$ such that

$$
\begin{gathered}
\forall s \in \omega\left(k_{r_{s}}=k^{*} \wedge a_{k^{*}}^{r_{s}+1} \mathcal{R}_{k^{*}} a_{k^{*}}^{r_{s}}\right) \\
\forall r \in \omega \backslash\left\{r_{s}: s \in \omega\right\} a_{k^{*}}^{r+1}=a_{k^{*}}^{r} .
\end{gathered}
$$

If $r \in \omega$ and $s^{*}:=\min \left\{s \in \omega: r_{s} \geq r\right\}$, then $r_{s^{*}} \geq r$ and by (16) and (17) we have $a_{k^{*}}^{r_{s^{*}+1}} \mathcal{R}_{k^{*}} a_{k^{*}}^{r_{s^{*}}}=a_{k^{*}}^{r_{s^{*}-1}}=a_{k^{*}}^{r_{s^{*}}-2}=\ldots=a_{k^{*}}^{r}$. This implies that the 


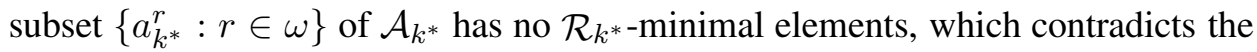
assumption that $\mathcal{W}_{k^{*}} \in \mathrm{Wfr}$.

(b) If $\mathbb{X}, \mathbb{Y} \in \mathcal{C}$ and $\mathbb{X} \preccurlyeq_{m} \mathbb{Y}$, then, by the assumption, for each $k<n$ we have $\theta_{k}(\mathbb{X})=\theta_{k}(\mathbb{Y})$ or $\theta_{k}(\mathbb{X}) \mathcal{R}_{k} \theta_{k}(\mathbb{Y})$. So, if $\theta_{k}(\mathbb{X})=\theta_{k}(\mathbb{Y})$, for all $k<n$, then by (14) we have $\theta(\mathbb{X})=\theta(\mathbb{Y})$. Otherwise, there is $k<n$ such that $\theta_{k}(\mathbb{X}) \mathcal{R}_{k} \theta_{k}(\mathbb{Y})$ and, by (13), $\theta_{k}(\mathbb{X}) \mathcal{R} \theta_{k}(\mathbb{Y})$. Statement (c) follows from (14) and (11).

Some examples of diagonal products Let LO, PO, Ord and Ord* denote the classes of linear orders, partial orders, ordinals and reversed ordinals respectively.

Fact 4.5 Let $\mathbb{X}, \mathbb{Y} \in \mathrm{PO}$.

(a) If $\mathbb{L} \in \mathrm{LO}$, then $\operatorname{Mono}(\mathbb{L}, \mathbb{X})=\operatorname{Emb}(\mathbb{L}, \mathbb{X})$;

(b) $\{\mathbb{L} \in \mathrm{LO}: \mathbb{L} \preccurlyeq m \mathbb{X}\}=\{\mathbb{L} \in \mathrm{LO}: \mathbb{L} \hookrightarrow \mathbb{X}\}$;

(c) If $\mathbb{X} \preccurlyeq_{m} \mathbb{Y}$, then $\{\mathbb{L} \in \mathrm{LO}: \mathbb{L} \hookrightarrow \mathbb{X}\} \subset\{\mathbb{L} \in \mathrm{LO}: \mathbb{L} \hookrightarrow \mathbb{Y}\}$.

Proof. (a) Let $f: \mathbb{L} \rightarrow \mathbb{X}$ be a monomorphism. If $x, y \in L$ and $f(x)<\mathbb{X} f(y)$, then $x \neq y$ and, since $f$ is a homomorphism, $y<x$ would imply $f(y)<\mathbb{X} f(x)$, which is not true. So, since $\mathbb{L}$ is a linear order, $x<y$, and, thus, $f$ is a strong homomorphism. Since $f$ is one-to-one it is an embedding. (b) follows from (a).

(c) Let $f: \mathbb{X} \rightarrow \mathbb{Y}$ be a monomorphism and let $g: \mathbb{L} \hookrightarrow \mathbb{X}$. Then $f\lceil g[L]$ is a monomorphism from the linear order $\left\langle g[L],<_{\mathbb{X}}\lceil g[L]\rangle\right.$ into the poset $\mathbb{Y}$ and, by (a) it is an embedding. Thus $(f\lceil g[L]) \circ g: \mathbb{L} \hookrightarrow \mathbb{Y}$.

Let the (class) functions $\theta_{0}, \theta_{1}: \mathrm{PO} \rightarrow$ Ord be defined by:

$\theta_{0}(\mathbb{X})=\sup \{\alpha \in$ Ord $: \alpha \hookrightarrow \mathbb{X}\}$ and $\theta_{1}(\mathbb{X})=\sup \left\{\alpha \in\right.$ Ord $\left.: \alpha^{*} \hookrightarrow \mathbb{X}\right\}$

Proposition 4.6 If (*) holds, $\mathbb{X}_{i} \in \mathrm{PO}$, for $i \in I$, and $\left\langle\left\langle\theta_{0}\left(\mathbb{X}_{i}\right), \theta_{1}\left(\mathbb{X}_{i}\right)\right\rangle: i \in I\right\rangle$ is a finite-to-one sequence, then $\bigcup_{i \in I} \mathbb{X}_{i}$ is a reversible poset.

Proof. If $\mathbb{X}, \mathbb{Y} \in \mathrm{PO}$ and $\mathbb{X} \preccurlyeq m \mathbb{Y}$, then by Fact 4.5 (c) $\{\alpha \in$ Ord $: \alpha \hookrightarrow \mathbb{X}\} \subset$ $\{\alpha \in$ Ord $: \alpha \hookrightarrow \mathbb{Y}\}$ and, hence, $\theta_{0}(\mathbb{X}) \leq \theta_{0}(\mathbb{Y})$. So $\theta_{0} \in \mathcal{M}($ PO, Ord $)$ and, similarly, $\theta_{1} \in \mathcal{M}$ (PO, Ord). By Theorem 4.4 (b) we have $\theta \in \mathcal{M}(\mathrm{PO}$, Ord $\times$ Ord $)$, where $\theta: \mathrm{PO} \rightarrow \operatorname{Ord} \times$ Ord is defined by $\theta(\mathbb{X})=\left\langle\theta_{0}(\mathbb{X}), \theta_{1}(\mathbb{X})\right\rangle$. Now the statement follows from Theorem 4.1

Example 4.7 Let $I$ be the set of pairs of countably infinite ordinals, that is $I=$ $\left(\omega_{1} \backslash \omega\right)^{2}$, and let $\mathbb{X}_{\langle\alpha, \beta\rangle}$, for $\langle\alpha, \beta\rangle \in I$, be disjoint partial orders such that, using the notation from Proposition 4.6, $\theta_{0}\left(\mathbb{X}_{\langle\alpha, \beta\rangle}\right)=\alpha$ and $\theta_{1}\left(\mathbb{X}_{\langle\alpha, \beta\rangle}\right)=\beta$. Then $\bigcup_{\langle\alpha, \beta\rangle \in I} \mathbb{X}_{\langle\alpha, \beta\rangle}$ is a reversible poset. If, in particular, $X_{\langle\alpha, \beta\rangle} \cong \beta^{*}+\alpha$, this follows from Corollary 5.3 as well. We note that here the sequences $\left\langle\theta_{0}\left(\mathbb{X}_{i}\right): i \in I\right\rangle$ and $\left\langle\theta_{1}\left(\mathbb{X}_{i}\right): i \in I\right\rangle$ are not finite-to-one, but $\left\langle\theta\left(\mathbb{X}_{i}\right): i \in I\right\rangle$ is one-to-one. 


\section{Applications: disjoint unions of chains}

$\sigma$-scattered chains We recall that a linear order (chain) $\mathbb{L}$ is called scattered, we will write $\mathbb{L} \in \mathrm{Scatt}$, if it does not contain a dense suborder (equivalently, iff $\mathbb{Q} \hookrightarrow \mathbb{L}$ ); $\mathbb{L}$ is said to be $\sigma$-scattered, we will write $\mathbb{L} \in \sigma$-Scatt, iff $\mathbb{L}$ is at most countable union of scattered linear orders.

Proposition 5.1 If $(*)$ holds and $\mathbb{X}_{i}, i \in I$, are $\sigma$-scattered linear orders, then

$\left\langle\left[\mathbb{X}_{i}\right]_{\rightleftarrows}: i \in I\right\rangle$ is a finite-to-one sequence $\mathbb{1 1}^{11} \Rightarrow \bigcup_{i \in I} \mathbb{X}_{i}$ is a reversible poset.

Proof. Clearly, $\langle\sigma$-Scatt, $\hookrightarrow\rangle$ is a preorder, the bi-embedability relation $\rightleftarrows$ defined on $\sigma$-Scatt by: $\mathbb{L} \rightleftarrows \mathbb{L}^{\prime} \Leftrightarrow \mathbb{L} \hookrightarrow \mathbb{L}^{\prime} \wedge \mathbb{L}^{\prime} \hookrightarrow \mathbb{L}$, is an equivalence relation, and, denoting the equivalence class of $\mathbb{L}$ by $[\mathbb{L}]_{\rightleftarrows}$, we obtain the corresponding antisymmetric quotient, $\langle\sigma$-Scatt $/ \rightleftarrows, \unlhd\rangle$, where the partial order $\unlhd$ is defined by $[\mathbb{L}]_{\rightleftarrows} \unlhd\left[\mathbb{L}^{\prime}\right]_{\rightleftarrows} \Leftrightarrow \mathbb{L} \hookrightarrow \mathbb{L}^{\prime}$. Writing $\mathbb{L} \prec \mathbb{L}^{\prime}$ iff $\mathbb{L} \hookrightarrow \mathbb{L}^{\prime} \wedge \mathbb{L}^{\prime} \hookrightarrow \mathbb{L}$, the corresponding strict (irreflexive) partial order is the structure $\langle\sigma$-Scatt $/ \rightleftarrows, \triangleleft\rangle$, where

$$
[\mathbb{L}] \rightleftarrows \triangleleft\left[\mathbb{L}^{\prime}\right]_{\rightleftarrows} \Leftrightarrow \mathbb{L} \prec \mathbb{L}^{\prime}
$$

From the classical Laver's result (that $\langle\sigma$-Scatt, $\hookrightarrow\rangle$ is a better-quasi-order, see [15, [16]) it follows that in the class $\sigma$-Scatt there are no decreasing sequences of the form $\mathbb{L}_{0} \succ \mathbb{L}_{1} \succ \mathbb{L}_{2} \succ \ldots$. Assuming that $\langle\sigma$-Scatt / $\rightleftarrows, \triangleleft\rangle \notin$ Wfr we would have a nonempty set $X \subset \sigma$-Scatt / $\rightleftarrows$, such that for each $y \in X$ there is $z \in X$ satisfying $z \triangleleft y$ and, hence, there would be a decreasing sequence $y_{0} \triangleright y_{1} \triangleright$ $y_{2} \triangleright \ldots$. Choosing $\mathbb{L}_{i} \in y_{i}$, for $i \in \omega$, we would obtain a sequence $\mathbb{L}_{0} \succ \mathbb{L}_{1} \succ$ $\mathbb{L}_{2} \succ \ldots$, which is impossible. So, $\langle\sigma$-Scatt $/ \rightleftarrows, \triangleleft\rangle \in$ Wfr.

Let $\theta: \mathcal{X} \rightarrow \sigma$-Scatt $/ \rightleftarrows$ be given by $\theta\left(\mathbb{X}_{i}\right)=\left[\mathbb{X}_{i}\right]_{\rightleftarrows}$. If $\mathbb{X}_{i} \preccurlyeq m \mathbb{X}_{j}$, then, since monomorphisms of linear orders are embeddings, $\mathbb{X}_{i} \hookrightarrow \mathbb{X}_{j}$, and, hence, $\left[\mathbb{X}_{i}\right] \unlhd\left[\mathbb{X}_{j}\right]$. Thus $(10)$ is true, $\theta \in \mathcal{M}(\mathcal{X})$, and we apply Theorem 4.1 $\left(I_{[\mathbb{L}] \rightleftarrows}^{\theta}=\right.$ $\left\{i \in I: \mathbb{X}_{i} \rightleftarrows \mathbb{L}\right\}, \mathbb{L} \in \sigma$-Scatt, are finite sets.)

Example 5.2 If $\mathbb{X}_{i}, i \in I$, are arbitrary linear orders of size $\leq \omega$ such that the sequence $\left\langle\left[\mathbb{X}_{i}\right]_{\rightleftarrows}: i \in I\right\rangle$ is finite-to-one, then $\bigcup_{i \in I} \mathbb{X}_{i}$ is a reversible poset. This follows from Proposition 5.1, since $\mathbb{L} \in \sigma$-Scatt, for each countable linear order $\mathbb{L}$. We note that, in that case, $\mathbb{X}_{i} \in$ Scatt, for all except finitely many $i \in I$ (because each countable non-scattered linear order is bi-embedable with $\mathbb{Q}$ ).

\footnotetext{
${ }^{11}$ that is, there is no infinite $J \subset I$ such that $\mathbb{X}_{i} \rightleftarrows \mathbb{X}_{j}$, for all $i, j \in J$.
} 
CSB chains of a limit type We recall that the order type of a linear order $\mathbb{L}$ is the class $\operatorname{otp}(\mathbb{L})=[\mathbb{L}] \cong$ of all linear orders isomorphic to $\mathbb{L}$. $\mathbb{L}$ will be called Cantor-Schröder-Bernstein (for embeddings) iff for each linear order $\mathbb{L}^{\prime}$ satisfying $\mathbb{L}^{\prime} \rightleftarrows \mathbb{L}$ we have $\mathbb{L}^{\prime} \cong \mathbb{L}$, that is, $[\mathbb{L}]_{\rightleftarrows}=[\mathbb{L}] \cong$. So, by Proposition 5.1 we have

Corollary 5.3 (*) If $\mathbb{X}_{i}, i \in I$, are $\sigma$-scattered CSB linear orders, then

$\left\langle\operatorname{otp}\left(\mathbb{X}_{i}\right): i \in I\right\rangle$ is a finite-to-one sequence $\mathbb{1 2}^{12} \Rightarrow \bigcup_{i \in I} \mathbb{X}_{i}$ is a reversible poset.

A scattered linear order $\mathbb{L}$ is said to be of a limit type iff $\mathbb{L} \rightleftarrows \sum_{s \in S} \mathbb{L}_{s}$, where $\mathbb{S} \in$ Scatt and $\mathbb{L}_{s} \cong \omega$ or $\mathbb{L}_{s} \cong \omega^{*}$, for each $s \in S 13$ For example, the linear order $\mathbb{L}=\omega \omega^{*}+1$ is of a limit type because $\mathbb{L} \rightleftarrows \omega \omega^{*}$ but, since $\mathbb{L} \not \omega \omega^{*}$, it is not CSB. Successor ordinals are CSB, but not of a limit type; limit ordinals are CSB of a limit type.

In order to describe CSB chains of a limit type let $\mathcal{W}$ denote the class of well orders, $\mathcal{L}$ the class of well orders isomorphic to limit ordinals, $\mathcal{Z}$ the class of linear orders isomorphic to $\omega^{\theta} \omega^{*}+\omega^{\delta}$, where $\theta$ and $\delta$ are ordinals satisfying $1 \leq \theta<\delta$. $\mathcal{W}^{*}, \mathcal{L}^{*}$, and $\mathcal{Z}^{*}$ will denote the classes of the inverses of elements of $\mathcal{W}, \mathcal{L}$, and $\mathcal{Z}$, respectively. (Clearly, $\left(\omega^{\theta} \omega^{*}+\omega^{\delta}\right)^{*}=\left(\omega^{\delta}\right)^{*}+\left(\omega^{\theta}\right)^{*} \omega$.)

Theorem 5.4 (a) A linear order is CSB of a limit type iff it is isomorphic to a finite sum of linear orders from $\mathcal{L} \cup \mathcal{L}^{*} \cup \mathcal{Z} \cup \mathcal{Z}^{*}$.

(b) If (*) holds and if $\mathbb{X}_{i}, i \in I$, are CSB linear orders of a limit type, then

$\bigcup_{i \in I} \mathbb{X}_{i}$ is a reversible poset $\Leftrightarrow\left\langle\operatorname{otp}\left(\mathbb{X}_{i}\right): i \in I\right\rangle$ is a finite-to-one sequence.

Proof. (a) From recent results of Laflamme, Pouzet, and Woodrow, (see [14]) it follows that a scattered linear order is CSB iff it is isomorphic to a finite sum of linear orders from $\mathcal{W} \cup \mathcal{W}^{*} \cup \mathcal{Z} \cup \mathcal{Z}^{*}$.

Let $\mathbb{L}$ be CSB linear order of a limit type, presented as a sum $\mathbb{L}=\mathbb{L}_{1}+\ldots+\mathbb{L}_{n}$, where $\mathbb{L}_{i} \in \mathcal{W} \cup \mathcal{W}^{*} \cup \mathcal{Z} \cup \mathcal{Z}^{*}$, for $i \leq n$. Let, in addition, this is a presentation of $\mathbb{L}$ with the minimal number of summands from $\mathcal{W} \cup \mathcal{W}^{*} \cup \mathcal{Z} \cup \mathcal{Z}^{*}$ (for example, $\omega+\omega \in \mathcal{W}$, but this linear order can be presented as a sum of finitely many elements of $\mathcal{W}$ in infinitely many ways).

Suppose that $i \leq n$ and $\mathbb{L}_{i} \in \mathcal{W} \backslash \mathcal{L}$, that is $\mathbb{L}_{i}=\mathbb{A}+\mathbb{B}$, where $\mathbb{A} \cong \gamma \in$ $\mathcal{L} \cup\{0\}, B=\left\{b_{0}, \ldots, b_{k-1}\right\}$, for some $k \in \mathbb{N}$, and $b_{0}<b_{1}<\ldots<b_{k-1}$. Since $\mathbb{L}$ is of a limit type, $b_{0}$ belongs to a convex part $C$ of $\mathbb{L}$ such that $C \cong \omega$ or $C \cong \omega^{*}$.

If $C \cong \omega$, then $C_{1}:=\left(b_{k-1}, \infty\right)_{\mathbb{L}} \cap C$ is a convex part of $\mathbb{L}$ of type $\omega$ and $b_{k}:=\min C_{1}=\min \mathbb{L}_{i+1}$, which implies that $\mathbb{L}_{i+1} \in \mathcal{W}$, because the linear

\footnotetext{
${ }^{12}$ that is, there is no infinite $J \subset I$ such that $\mathbb{X}_{i} \cong \mathbb{X}_{j}$, for all $i, j \in J$.

${ }^{13} \mathbb{L} \in$ Scatt is of a limit type iff $\mathbb{L}$ has no points which are left fixed under every $f \in \operatorname{Emb}(\mathbb{L})$, iff no 1 appears in the expression of $\mathbb{L}$ as a minimal sum of hereditarily additively indecomposable linear orders; see [16], p. 112.
} 
orders from $\left(\mathcal{Z} \cup \mathcal{Z}^{*} \cup \mathcal{W}^{*}\right) \backslash \mathcal{W}$ have no minimum. Thus $\mathbb{L}_{i}+\mathbb{L}_{i+1} \in \mathcal{W}$, which contradicts the minimality of $n$.

If $C \cong \omega^{*}$, then $\gamma=0, i>1, \mathbb{L}_{i-1} \notin \mathcal{Z} \cup \mathcal{Z}^{*}$ (since the linear orders from $\mathcal{Z} \cup \mathcal{Z}^{*}$ do not have a largest element) and, hence, $\mathbb{L}_{i-1} \in \mathcal{W}^{*}$ and $\mathbb{L}_{i-1}+\mathbb{L}_{i} \in \mathcal{W}^{*}$, which contradicts the minimality of $n$ again.

So, $\mathbb{L}_{i} \in \mathcal{W}$ implies that $\mathbb{L}_{i} \in \mathcal{L}$ and, similarly, $\mathbb{L}_{i} \in \mathcal{W}^{*}$ implies that $\mathbb{L}_{i} \in \mathcal{L}^{*}$.

Conversely, let $\mathbb{L}=\mathbb{L}_{1}+\ldots+\mathbb{L}_{n}$, where $\mathbb{L}_{i} \in \mathcal{L} \cup \mathcal{L}^{*} \cup \mathcal{Z} \cup \mathcal{Z}^{*}$, for $i \leq n$, and $n$ is the minimal number of summands. It is well known that the binary relation $\sim$ on $L$ defined by: $x \sim y$ iff $|[\min \{x, y\}, \max \{x, y\}]|<\omega$, is an equivalence relation and (see [17], p. 71) $\mathbb{L}=\sum_{t \in T} \mathbb{L}_{t}$, where $\mathbb{T} \in$ Scatt, $\left\{L_{t}: t \in T\right\}=L / \sim$ and $\operatorname{otp}\left(\mathbb{L}_{t}\right) \in \mathbb{N} \cup\left\{\omega, \omega^{*}, \zeta\right\}$, for each $t \in T$ (where $\zeta:=\operatorname{otp}(\mathbb{Z})$ ). So, since each $x \in L$ belongs to a convex subset $C$ of $L$, which is either isomorphic to $\omega$ (if $x \in L_{i}$ and $\mathbb{L}_{i} \in \mathcal{L} \cup \mathcal{Z}$ ) or to $\omega^{*}$ (if $x \in L_{i}$ and $\mathbb{L}_{i} \in \mathcal{L}^{*} \cup \mathcal{Z}^{*}$ ), we have $\operatorname{otp}\left(\mathbb{L}_{t}\right) \notin \mathbb{N}$, for all $t \in T$. Thus $\mathbb{L}$ can be presented as a sum of linear orders isomorphic to $\omega, \omega^{*}$, or $\zeta=\omega^{*}+\omega$ and, hence, it is of a limit type.

(b) The implication " $\Leftarrow$ " follows from Corollary 5.3 .

Suppose that the sequence $\left\langle\operatorname{otp}\left(\mathbb{X}_{i}\right): i \in I\right\rangle$ is not finite-to-one and $J=$ $\left\{i_{k}: k \in \omega\right\} \subset I$, where $i_{k} \neq i_{l}$ and $\mathbb{X}_{i_{k}} \cong \mathbb{X}_{i_{l}}$, for different $i, j \in J$. By the assumptions we have $\mathbb{X}_{i_{0}} \cong \sum_{s \in S} \mathbb{L}_{s}$, where $\mathbb{L}_{s} \cong \omega$ or $\mathbb{L}_{s} \cong \omega^{*}$. For $s \in S$ let $L_{s}=\left\{a_{0}^{s}, a_{1}^{s}, a_{2}^{s}, \ldots\right\}$ be an enumeration such that $a_{0}^{s}<a_{1}^{s}<a_{2}^{s}<\ldots$, if $\mathbb{L}_{s} \cong \omega$, and $a_{0}^{s}>a_{1}^{s}>a_{2}^{s}>\ldots$, if $\mathbb{L}_{s} \cong \omega^{*}$. Then defining $A_{0}:=\left\{a_{2 n}^{s}: s \in S \wedge n \in \omega\right\}$ and $A_{1}:=\left\{a_{2 n+1}^{s}: s \in S \wedge n \in \omega\right\}$ we have $\mathbb{A}_{0} \cong \mathbb{A}_{1} \cong \mathbb{X}_{i_{0}}$ and $\left\{A_{0}, A_{1}\right\}$ is a partition of $X_{i_{0}}$. Let $f \in \operatorname{Sur}(I) \backslash \operatorname{Sym}(I)$ be defined by $f\left(i_{0}\right)=f\left(i_{1}\right)=i_{0}$, $f\left(i_{k}\right)=i_{k-1}$, for $k \in \mathbb{N}$, and $f(i)=i$, for $i \in I \backslash\left\{i_{k}: k \in \omega\right\}$. Then $X_{i_{0}}$ is partitioned into copies of $X_{i_{0}}$ and $X_{i_{1}}$ and, by Corollary 2.6, the poset $\bigcup_{i \in I} \mathbb{X}_{i}$ is not reversible.

We note that, in particular, Theorem 5.4(b) gives a characterization of reversibility in the class of posets of the form $\mathbb{X}=\bigcup_{i \in I} \mathbb{X}_{i}$, where $\mathbb{X}_{i} \in \mathcal{L} \cup \mathcal{L}^{*}$, for $i \in I$. The corresponding characterization, when $\mathbb{X}_{i} \in \mathcal{W} \cup \mathcal{W}^{*}$, for $i \in I$, is given in [12].

Acknowledgments This research was supported by the Ministry of Education and Science of the Republic of Serbia (Project 174006).

\section{References}

[1] B. Dushnik, E. W. Miller, Concerning similarity transformations of linearly ordered sets, Bull. Amer. Math. Soc. 46 (1940) 322-326.

[2] M. Kukieła, Reversible and bijectively related posets, Order 26 (2009) 119-124. 
[3] M. Kukieła, Characterization of hereditarily reversible posets, Math. Slovaca 66,3 (2016) 539544.

[4] M. S. Kurilić, From $A_{1}$ to $D_{5}$ : Towards a forcing-related classification of relational structures, J. Symbolic Logic 79,1 (2014) 279-295.

[5] M. S. Kurilić, Retractions of reversible structures, J. Symbolic Logic, (in print).

[6] M. S. Kurilić, Reversibility of definable relations, (to appear)

[7] M. S. Kurilić, Copy-minimal and embedding-rigid structures, (to appear).

[8] M. S. Kurilić, N. Morača, Condensational equivalence, equimorphism, elementary equivalence and similar similarities, Ann. Pure Appl. Logic 168,6 (2017) 1210-1223.

[9] M. S. Kurilić, N. Morača, Reversibility of extreme relational structures, (to appear)

[10] M. S. Kurilić, N. Morača, Reversible sequences of cardinals, reversible equivalence relations, and similar structures, (to appear) https://arxiv.org/abs/1709.09492

[11] M. S. Kurilić, N. Morača, Variations of reversibility, (to appear)

[12] M. S. Kurilić, N. Morača, Reversible disjoint unions of well orders and their inverses, (to appear)

[13] A. H. Lachlan, R. E. Woodrow, Countable ultrahomogeneous undirected graphs, Trans. Amer. Math. Soc., 262,1 (1980) 51-94.

[14] C. Laflamme, M. Pouzet, R. Woodrow, Equimorphy: the case of chains, Arch. Math. Logic 56, 7-8 (2017) 811-829.

[15] R. Laver, On Fraïssé's order type conjecture, Ann. of Math. 93,2 (1971) 89-111.

[16] R. Laver, An order type decomposition theorem Ann. of Math. 98,1 (1973) 96-119.

[17] J. G. Rosenstein, Linear orderings, Pure and Applied Mathematics, 98, Academic Press, Inc., Harcourt Brace Jovanovich Publishers, New York-London, 1982.

[18] J. H. Schmerl, Countable homogeneous partially ordered sets, Algebra Univers. 9,3 (1979) 317-321.

[19] P. Vopěnka, A. Pultr, Z. Hedrlín, A rigid relation exists on any set, Comment. Math. Univ. Carolinae 6 (1965) 149-155. 